\title{
The shape of the cosmic ray proton spectrum
}

\author{
Paolo Lipari ${ }^{1}$ and Silvia Vernetto ${ }^{2}$ \\ ${ }^{1}$ INFN, Sezione Roma "Sapienza", Piazzale Aldo Moro 2, 00185 Roma, Italy \\ ${ }^{2}$ OATO-INAF, INFN Sezione Torino, Via Pietro Giuria 1, 10124 Torino, Italy
}

4th November 2019

Keywords - Cosmic Rays, Protons, Extensive Air Showers

\begin{abstract}
Recent observations of cosmic ray protons in the energy range $10^{2}-10^{5} \mathrm{GeV}$ have revealed that the spectrum cannot be described by a simple power law. A hardening of the spectrum around an energy of order few hundred $\mathrm{GeV}$, first observed by the magnetic spectrometers PAMELA and AMS02, has now been confirmed by several calorimeter detectors (ATIC, CREAM, CALET, NUCLEON and DAMPE). These new measurements reach higher energy and indicate that the hardening corresponds to a larger step in spectral index than what estimated by the magnetic spectrometers. Data at still higher energy (by CREAM, NUCLEON and DAMPE) show that the proton spectrum undergoes a marked softening at $E \approx 10^{4} \mathrm{GeV}$. Understanding the origin of these unexpected spectral features is a significant challenge for models of the Galactic cosmic rays. An important open question is whether additional features are present in the proton spectrum between the softening and the "Knee". Extensive Air Shower detectors, using unfolding procedures that require the modeling of cosmic ray showers in the atmosphere, estimated the proton flux below and around the Knee (at $E \simeq 3 \mathrm{PeV}$ ). These results however have large systematic uncertainties and are in poor agreement with each other. The measurement in the PeV energy range, recently presented by IceTop/IceCube, indicates a proton flux higher than extrapolations of the direct measurements calculated assuming a constant slope, and therefore requires the existence of an additional spectral hardening below the Knee. A clarification of this point is very important for an understanding of the origin of the Galactic cosmic rays, and is also essential for a precise calculation of the spectra of atmospheric neutrinos in the energy range $(E \gtrsim 10 \mathrm{TeV})$ where they constitute the foreground for the emerging astrophysical $\nu$ signal.
\end{abstract}

\section{Introduction}

The precise measurement of the cosmic ray (CR) spectra is a fundamental task for high energy astrophysics, because they encode important information about their sources and the properties of their propagation in the Galaxy. In first approximation, the spectra of protons and primary nuclei, in a broad energy range that extends from approximately $30 \mathrm{GeV}$ to the so called "Knee" at energy few PeV's, have a power-law form. Recent observations have however revealed that the spectra deviate from this simple form, and contain additional structures. In particular, direct measurements of the proton flux show the existence of two spectral features: a hardening at $E \approx 500 \mathrm{GeV}$, and a softening at $E \approx 10^{4} \mathrm{GeV}$.

In this work we review the evidence for these features and discuss the possible existence of additional spectral structures between $10 \mathrm{TeV}$ and the Knee, extrapolating the results of the direct measurements of the proton flux to higher energy, where estimates of the flux have been obtained by Extensive Air Shower (EAS) detectors.

Our discussion in the present work will be limited to protons. This is because they are the largest component of the CR flux and their spectral shape is measured more accurately. The comparison of the spectra of different particle types is of course of great interest. Several models for the origin of Galactic cosmic rays predict 
that the spectra of protons and primary nuclei (such as helium, carbon, oxygen, ...) have, at least in good approximation, the same spectral shape when expressed as a function of rigidity, and it is clearly very important to study experimentally the validity of this assumption. A discussion of the spectra of CR nuclei will be however postponed to a future publication.

This work is organized as follows. In Sec. 2 we analyze the direct (magnetic and calorimetric) measurements of the $p$ flux above $100 \mathrm{GeV}$, and discuss the evidence for the existence and the properties of the two (hardening and softening) features. In the following section we compare the extrapolations of a fit to the direct measurements with the published estimates of the proton spectrum obtained by EAS detectors. In a final section we summarize the results and give some conclusions.

\section{Measurements of the CR proton spectrum}

The existence of a hardening in the energy spectrum of protons (and other CR species) was first inferred in a paper of the CREAM Collaboration [1] from a comparison of their measurements of the spectral indices at $E \gtrsim 1 \mathrm{TeV}$, with the results obtained by magnetic spectrometers at lower energy $(E \sim 100 \mathrm{GeV})$. The flatter spectra observed at high energy suggested the existence of a "discrepant hardening" in the energy range $(0.2-1 \mathrm{TeV})$ where data were not yet available.

Hardenings in the proton and helium spectra were then directly observed by PAMELA [2, that reported the existence of "sharp" breaks at rigidity $\left(\rho_{p}=232_{-30}^{+35} \mathrm{GV}\right.$ for protons and $\rho_{\mathrm{He}} \simeq 243_{-31}^{+27} \mathrm{GV}$ for helium). At the break rigidity the proton flux was observed to flatten from a spectral index $\alpha_{1} \simeq 2.85 \pm 0.04$ to an index $\alpha_{2} \simeq 2.67 \pm 0.06$. These results generated considerable interest because simple and commonly accepted models for Galactic cosmic rays predicted spectra with the shape of an unbroken power law (above the maximum energy where solar modulations have a significant effect and up to the Knee at $E \simeq 3 \mathrm{PeV}$ ).

The existence of the hardenings for protons and helium nuclei was then confirmed, with higher statistical significance, by the AMS02 detector [3, 4. The spectral hardening for protons measured by AMS02 is centered at higher rigidity $\left(\rho_{p} \simeq 336_{-52}^{+95} \mathrm{GV}\right)$ in comparison to the PAMELA results, and has a smaller step in spectral index $\Delta \alpha \simeq 0.133_{-0.037}^{+0.056}$ (with $\alpha_{1} \simeq 2.849_{-0.005}^{+0.006}$, and $\alpha_{2} \simeq 2.716_{-0.056}^{+0.037}$ ).

Measurements of the proton spectrum above $1 \mathrm{TeV}$ have been now obtained by several CR calorimeters: ATIC [5], CREAM [6], CALET [7, NUCLEON [8, 9] and DAMPE [10]. The ATIC, CREAM and NUCLEON detectors cover only the energy range $E \gtrsim 1 \mathrm{TeV}$, and therefore do not observe directly the hardening, but only the spectral shape above it. The CALET and DAMPE instruments measure the proton spectrum across the entire region of the hardening.

Three detectors (CREAM, NUCLEON and DAMPE) reach a maximum energy of order $100 \mathrm{TeV}$, and they all have indications (or clear evidence in the case of DAMPE) for the existence of a second spectral feature: a softening centered at an energy of order $10 \mathrm{TeV}$.

The measurements of the proton flux listed above are shown in Fig. 1 plotted in the form $E^{2.7} \phi(E)$ versus the kinetic energy $E$ to enhance the visibility of the spectral features. The error bars of the PAMELA and AMS02 data points shown in the figure are calculated combining quadratically statistical and systematic errors. For the other detectors they only show statistical uncertainties. For CREAM, CALET and DAMPE the systematic error (mainly due to the calibration of the energy scale) is of order 10\%. For ATIC we estimated a systematic error of $20 \%$. For the NUCLEON detector the spectrum given in the figure is the one obtained estimating the energy with the kinematical method KLEM (Kinematic Lightweight Energy Meter), and the systematic error is of order $25 \%$.

Taking into account the systematic errors, all the measurements in Fig. 1 are in reasonable good agreement in the energy ranges where they overlap (see more discussion below). A visual inspection of the figure immediately shows that a simple power-law cannot give a good description of the observations, and both the hardening (at $E \approx 500 \mathrm{GeV}$ ) and the softening (around $E \approx 10-20 \mathrm{TeV}$ ) are clearly visible.

To study more quantitatively the spectral shape of the proton flux we have fitted the data using the "two- 
break" expression:

$$
\phi(E)=K\left(\frac{E}{E_{0}}\right)^{-\alpha_{1}}\left[1+\left(\frac{E}{E_{b}}\right)^{1 / w}\right]^{-\left(\alpha_{2}-\alpha_{1}\right) w}\left[1+\left(\frac{E}{E_{b}^{\prime}}\right)^{1 / w^{\prime}}\right]^{-\left(\alpha_{3}-\alpha_{2}\right) w^{\prime}}
$$

where $E_{0}$ is an arbitrary reference energy, $K$ gives the absolute normalization, and $\left\{\alpha_{1}, E_{b}, \alpha_{2}, w, E_{b}^{\prime}, \alpha_{3}, w^{\prime}\right\}$ are free parameters that determine the spectral shape. The quantities $E_{b}$ and $E_{b}^{\prime}$ are the "break" energies around which the spectral index changes, all other parameters are adimensional. Without loss of generality one can impose the constraints $E_{b}<E_{b}^{\prime}$, and $w$ and $w^{\prime}$ positive.

Expression (1) is an obvious generalization of the "one-break" form:

$$
\phi(E)=K\left(\frac{E}{E_{0}}\right)^{-\alpha_{1}}\left[1+\left(\frac{E}{E_{b}}\right)^{1 / w}\right]^{-\left(\alpha_{2}-\alpha_{1}\right) w}
$$

that describes a flux with a single spectral feature centered at the energy $E_{b}$, where the spectral index changes from $\alpha_{1}$ (for $E \ll E_{b}$ ) to $\alpha_{2}$ (for $E \gg E_{b}$ ). The parameter $w$ gives the width in $\log E$ of the step in spectral index with $w=0$ corresponding to a discontinuous variation. The relation between the parameter $w$ and the width in $\log E$ is discussed in detail in [11. A useful expression for this relation is:

$$
\left(\Delta \log _{10} E\right)_{\Delta \alpha / 2}=\left(\log _{10} 9\right) w=0.954 w .
$$

This equation states that one half of the step in spectral index develops in an interval of $\log _{10} E$ approximately equal to $w$.

In the two-break expression of Eq. (1) the spectrum has slope $\alpha_{1}$ for $E \ll E_{b}$ and $\alpha_{3}$ for $E \gg E_{b}^{\prime}$, while in the interval $E_{b}<E<E_{b}^{\prime}$ the spectral index is approximately equa ${ }^{1}$ to $\alpha_{2}$.

The generalization of expression (1) for a spectrum that contains an arbitrary number of features is obvious, and simply requires the inclusion of a new distortion factor for each feature. Each factor (in square parenthesis in the formula) depends on three parameters $\left\{E_{b, j}, \Delta \alpha_{j}, w_{j}\right\}$ that give the position, size and width of the $j$-th feature.

The family of curves described by Eq. (2) has been used to fit "features" in the CR spectra (including the proton flux) in several papers, using however different parametrizations. The main source of potential confusion is the choice for the parameter that describes how "gradual" is the step in spectral index. AMS02 3], CALET [7] and DAMPE [10] adopted the parameter $s=w\left(\alpha_{2}-\alpha_{1}\right)$, while NUCLEON [8] uses the parameter $S=1 / w$. The choice made here is motivated by the fact that the parameter $w$ has a simple and clear physical meaning as the width of the interval in $\log E$ where the spectral index undergoes its step, independently from the value of $\Delta \alpha$ [see Eq. (3)].

To study the shape of the proton spectrum and investigate how consistent are the different measurements we have performed separate fits to the data of the individual experiments. The DAMPE data [10] cover an energy interval that includes both spectral features, and have been fitted with the two-break expression of Eq. (1). The data of all other detectors cover an energy interval that includes only one of the two features (the lower energy hardening for PAMELA, AMS02, ATIC and CALET, and the higher energy softening for CREAM and NUCLEON), and have then been fitted with the single break expression of Eq. (2), using the appropriate and obvious renaming of the parameters when needed (for CREAM and NUCLEON).

The best fit spectra are shown in Fig. 1 and the parameters of the fits are listed in Table 1. Our results are consistent with the fits reported in the original publications.

The fits to the PAMELA and AMS02 data are performed combining quadratically statistical and systematic uncertainties. For the other experiments we use only statistical uncertainties. In these experiments the systematic errors for different points are strongly correlated and the fits performed with only statistical uncertainties give very good values for $\chi_{\min }^{2} / N_{\text {dof }}$ (see Table 1) (while fits using also systematic errors and neglecting correlations gives too small values of order $0.01-0.03)$.

${ }^{1}$ This is the case when the ratio $E_{b}^{\prime} / E_{b}$ is sufficiently large in comparison to the widths of the two features. That is when $\log _{10}\left(E_{b}^{\prime} / E_{b}\right) \gtrsim\left(w+w^{\prime}\right)$. 
Three data sets (DAMPE, NUCLEON and CREAM) show evidence for the existence of a spectral softening and weaker indications for this feature are also present in the ATIC and CALET data.

It should be noted that the CREAM Collaborations in [6] does not claim the detection of a softening feature, and fits the data taken in the energy interval $[1,200] \mathrm{TeV}$ with a simple unbroken power law obtaining a the spectral index $\alpha \simeq 2.61 \pm 0.01$. The CREAM paper discusses the possibility of a deviation from the power law form stating: "The spectra become softer above $\sim 20 \mathrm{TeV}$. However, our statistical uncertainties are large at these energies and more data are needed". It is nonetheless interesting to investigate quantitatively how strong is the indication of a softening. Fits of the CREAM data (that use only statistical errors) for a simple power law and with the one-break expression of Eq. (2) gives $\chi_{\min }^{2} \simeq 13.3$ and 1.9 respectively. Taking into account the different number of free parameters in the two cases, this corresponds to an indication with a significance of order $3.0 \sigma$ in favor of the existence of a softening.

We also performed a global fit of the data of all seven experiments in the energy range $E>50 \mathrm{GeV}$ using expression (1). The resulting best fit (obtained combining quadratically statistical and systematic uncertainties) is shown (as a thick red line) in Fig. 1. and the parameters are given in the right-hand column of Table 1 . The fit is of good quality, with $\chi_{\min }^{2}=46.5$ for 116 d.o.f.

From this study we can draw the following conclusions:

- In the energy range below the hardening the $p$ spectrum has been very accurately measured by the magnetic spectrometers AMS02 and PAMELA that obtain best fits to the spectral index that are nearly identical $\left(2.850 \pm 0.043\right.$ for PAMELA [2] and 2.849 ${ }_{-0.005}^{+0.006}$ for AMS02 [3]). The data of the calorimeter experiments (ATIC, CALET and DAMPE) are consistent with these results, even if the best fit of DAMPE indicates a slightly harder spectrum (with a slope given as $2.772 \pm 0.002$ in [10, while our fit yields $2.750 \pm 0.005$ ).

- The hardening of the spectrum for an energy (or rigidity) of order few hundred GeV is now very well established, but the precise shape of the feature is not well determined because the measurements of different experiments are not in perfect agreement with each other. The uncertainties are associated to all the three parameters that describe the shape of the feature: break energy, spectral index step $\Delta \alpha$ (or equivalently the slope $\alpha_{2}$ above the hardening) and width $w$. The slope $\alpha_{2}$ of the proton spectrum in the energy range $1-10 \mathrm{TeV}$ has now been measured by five different calorimeter experiments (ATIC, CREAM, CALET, DAMPE, and NUCLEON). For all these experiments the spectrum in the $1-10 \mathrm{TeV}$ energy range is harder ( $\alpha_{2}$ between 2.44 to 2.62$)$ than what is indicated by the AMS02 data $\left(\alpha_{2} \simeq 2.716_{-0.056}^{+0.037}[\underline{3}]\right)$. The most natural explanation for this discrepancy is that the AMS02 data, that are limited to a maximum rigidity of $1.2 \mathrm{TV}$, do not measure well the entire "development" of the hardening. Our fit to the combined data of all experiments yields a good quality fit $\left(\chi_{\min }^{2} / N_{\text {dof }} \simeq 0.40\right)$ with a gradual $(w \simeq 0.27 \pm 0.19)$ hardening centered at $E_{b} \simeq 650_{-150}^{+260} \mathrm{GeV}$ with a spectral index at high energy $\alpha_{2}=2.57_{-0.06}^{+0.04}$.

- The spectrum of protons exhibits a second spectral feature, a softening at $E \simeq 10-20 \mathrm{TeV}$. This conclusion is now supported by the observations of three experiments: CREAM, NUCLEON and DAMPE (with weaker indications in the data of ATIC and CALET). The evidence of DAMPE is the strongest, with a statistical significance estimated as $4.7 \sigma$ in [10]. the simpler analysis performed by us using only the statistical errors gives a very large $\Delta \chi^{2}=143$ between the two-break fit shown in Fig. 1 and one-break fit that extends as a simple power law above the hardening (corresponding nominally to more than $11 \sigma$ ). For the CREAM and NUCLEON data the existence of a spectral softening has a statistical significance of order $3 \sigma$.

\section{Air Shower measurements of the proton flux}

A question that emerges immediately from our discussion on the direct measurements of the CR proton flux is the shape of the spectrum at higher energy (in the range $10-10^{3} \mathrm{TeV}$ ). Does the spectrum continues as an unbroken power law up to the Knee or does it contain additional structures?

At present, direct measurements of the CR spectra extend only to a maximum energy of order $E \simeq 100 \mathrm{TeV}$, but information on the spectra at higher energy can be obtained from observations of the Extensive Air Showers 
(EAS) generated by primary cosmic rays in the Earth's atmosphere. These measurements are however model dependent because they require a comparison of the data with Montecarlo simulations of the shower development. The main uncertainty arises from our poor knowledge of the properties of hadronic interactions (such as multiplicity distributions and inclusive energy spectra of the particles in the final state of a collision).

Several experiments have published results on the all-particle spectrum, that sums the contributions of all CR components at the same energy per particle:

$$
\phi_{\text {all }}(E)=\sum_{\mathrm{A}} \phi_{\mathrm{A}}(E)
$$

Fig. 2 shows the all-particle spectrum measured by the Tibet detector 12 in the energy range $10^{5}-10^{7} \mathrm{GeV}$ and by the IceTop/IceCube detector [13] in the energy range $10^{7}-10^{8} \mathrm{GeV}$. For the Tibet experiment the figure gives three estimates of the spectrum that differ by approximately $\pm 10 \%$, obtained in [12] using different assumptions about the CR composition and different Montecarlo codes to model the hadronic interactions. For the IceTop/IceCube measurement the estimated systematic error (shown as a shaded area) is also of order $10 \%$. Taking into account these systematic uncertainties, the results are in good agreeement. Other measurements of the all-particle spectrum (for a review see [14]), including those of Kascade [15, are also consistent. The all-particle spectrum around $E \approx 3 \mathrm{PeV}$ exhibits the well-known and much discussed softening called the Knee, whose origin remains controversial and poorly understood.

The identification of the mass of the primary particlea and the measurement of the spectra of individual CR components is a more difficult task, with larger systematic uncertainties. The published estimates of the proton spectrum obtained by EAS detectors are shown in Fig. 2 .

In 2005 the Kascade Collaboration [15] published an "unfolding" of the CR flux as the sum of five mass components ( $p, \mathrm{He}, \mathrm{C}, \mathrm{Mg}, \mathrm{Fe}$ ). In the Kascade paper the unfolding is performed using two different Montecarlo codes to model high energy hadronic interactions: QGSJet-01 [16] and Sibyll 2.1 [17] (in both cases low energy interactions were modeled using the GHEISHA code [18]). The proton spectra estimated with these two methods (shown as diamonds and squares in Fig. 2) have similar shapes but their normalizations differ by approximately a factor of two for energies below the Knee $(E \simeq 1-2 \mathrm{PeV})$, so that the proton component accounts of approximately $30 \%$ of the all particle flux in one case, and only $15 \%$ in the other.

In 2013 the Kascade-Grande Collaboration [19] published a reanalysis of the Kascade data based on improved methods, and using as Montecarlo code for high energy interactions a new version of the QGSJet code [20], and at low energy the FLUKA code 21. The unfolded proton spectrum is shown as hexagons in Fig. 2, In this case the proton knee has a more gradual shape with a normalization that is similar to the one obtained using the Sibyll code in the previous analysis. The total systematic uncertainty estimated for this analysis [19] is shown as the shaded area.

An estimate of the proton flux at very high energy has also been obtained by the IceTop/IceCube Collaboration [13], unfolding the spectrum into four components ( $p$, helium, oxygen and iron). The proton flux from this analysis is shown in Fig. 2 as circles. In this study, the Montecarlo code used to model the development of high energy showers is Sibyll 2.1, also used by Kascade in [15, however the normalization of the proton spectrum is now much higher (for energy around $2 \mathrm{PeV}$ the difference in normalization is approximately a factor of three).

The reasons for such large differences between estimates of the proton flux in the PeV energy range are not clear, and this question certainly deserves a detailed study.

The large uncertainties about the $\mathrm{PeV}$ proton flux make the extrapolation of the direct measurements ambiguous. Different extrapolations can be constructed to agree with PeV data. This is illustrated in Fig. 2 that shows two possible extrapolations models.

One model (shown as the dashed line) assumes that the proton spectrum continues with a constant spectral index above the softening at $E \approx 15 \mathrm{TeV}$. With this assumption the extrapolation is in reasonable agreement with the estimate of Kascade-2013 [19.

A second model (shown as the dot-dashed line) is constructed to agree with the estimate of the proton measurement obtained by IceTop/IceCube. In order to do so, it is necessary to introduce additional structures in the spectrum. To connect the measured proton flux at $E_{1} \simeq 20 \mathrm{TeV}$ (where good precision direct measurements 
are available) to the IceTop/IceCube data at $E_{2} \simeq 2 \mathrm{PeV}$, one needs an average spectral index

$$
\left\langle\alpha\left(E_{1}, E_{2}\right)\right\rangle=-\frac{\ln \left(\phi_{2} / \phi_{1}\right)}{\ln E_{2} / E_{1}}
$$

Substituting in this expression the measured values one obtains the estimate $\langle\alpha\rangle \simeq 2.66 \pm 0.04$, where the error is calculated assuming a $15 \%$ error on each flux measurement. This value is significantly smaller than the slope measured with direct observations of the proton flux above $15 \mathrm{TeV}\left(\alpha_{3} \simeq 2.87\right)$. One must then conclude that if the IceTop/IceCube (and also the direct) measurements are correct, then the proton spectrum cannot have a constant spectral index in the energy interval $\left[E_{1}, E_{2}\right]$, and must contain a hardening. These considerations obviously do not allow to predict the detailed form of the spectrum, and the dot-dashed line shown in Fig. 2 is only one possible example.

\section{Discussion}

Precise measurements of the CR proton flux in the energy range $10^{2}-10^{5} \mathrm{GeV}$ have shown that the spectrum deviates from the simple power law shape predicted by commonly accepted models and exhibits two features. The first one is the sub- $\mathrm{TeV}$ hardening discovered by PAMELA, then observed by AMS02 and several calorimeter detectors. Measurements in the multi-TeV energy range indicate that the step in spectral index of the hardening is quite large, with slopes of order 2.85 and 2.60 below and above the break energy.

A second feature, a softening, has now been clearly detected at an energy of order $10 \mathrm{TeV}$ by DAMPE, confirmining the indications already present in the CREAM and NUCLEON observations. Taking into account the systematic uncertainties the measurements of these three detectors are in good agreement, with absolute fluxes that differ by approximately $10 \%$ around $E \simeq 10 \mathrm{TeV}$. Our global fit gives a break energy with a rather large error $E_{b}^{\prime}=16_{-8}^{+13} \mathrm{TeV}$. The slopes measured above the softening are also consistent (see Table 1). Our fit to all data above the softening gives a spectral index $2.87_{-0.10}^{+0.15}$.

There is clear evidence that the hardening is also present in the spectra of helium and other primary nuclei 2, 4, 22, and the results are consistent with the hypothesis that the feature has a "universal" shape when studied as a function of rigidity (even if the values of the slopes of different particle types are not equal).

The existing measurements of helium and other nuclei at $E \gtrsim 10 \mathrm{TeV}$ [6, 8, 9] suggest that the softening is also present for cosmic ray particles heavier than protons, and within the large statistical and systematic errors, the feature is again consistent with having a rigidity dependent shape, common to all particle types. More data are however required to reach a firm conclusion.

It is also interesting to note that the HAWC measurement of the all-particle spectrum [23] in the 10-500 TeV energy range shows a spectral break at the energy $45.7 \pm 0.1 \mathrm{TeV}$ where the index is oberved to change from $2.49 \pm 0.01$ to $2.71 \pm 0.01$ (with a step of approximately the same size as the one observed for the proton flux). Evidence for a strong break in the all-particle spectrum at a rigidity $7-17$ TV has also been reported by the NUCLEON experiment [8, 9].

Observations of the "light component" (proton and helium) of the CR spectrum in the energy range 5$200 \mathrm{TeV}$ have been performed by the EAS detector ARGO-YBJ 24] reporting a spectrum consistent with an unbroken power law. A subsequent measurement of the light component of the CR spectrum by ARGO-YBJ at higher energy has given indications for an intriguing "cutoff" at $E \approx 640 \pm 87 \mathrm{GeV}[25]$.

For an understanding of the origin of the shape of the proton spectrum it is very important to establish if additional spectral structures exist below the Knee. For this purpose it would be clearly desirable to extend the direct measurements to higher energy. At present measurements of the CR spectra at very high energy have been obtained by EAS detectors. This is a very difficult task, and the results are not in good agreement with each other. Estimates of the proton flux in the PeV energy range performed by Kascade and IceTop/IceCube for $E \simeq 2 \mathrm{PeV}$ differ by a factor larger than three.

These discrepancies do not allow to extrapolate the direct measurements of the proton flux in a unique way. Low estimates of the flux in the PeV energy range (such as those presented by Kascade in [19]) are consistent with the hypothesis that the $p$ spectrum continues with a constant index from the softening break at $\sim 15 \mathrm{TeV}$ 
up to the Knee. On the other hand, the higher flux recently obtained by IceTop/IceCube [13] requires the existence of additional spectral structures (with a new hardening).

Future EAS observations by detectors with the capability of measuring several components (electromagnetic, muonic, Cherenkov light, ...) of the showers (such as LHAASO 26] ) have the potential to significantly reduce the uncertainties in the measurement of the spectra for different primary particles.

The precise description of the CR spectra below the Knee is crucial for the calculation of the spectra of atmospheric neutrinos in the critical energy range $\left(E_{\nu} \sim 10-100 \mathrm{TeV}\right)$ where one observes the transition between a flux dominated by the softer atmospheric component to a flux dominated by astrophysical particles.

The discovery of the softening in the proton spectrum can have very important consequences for this problem. The models of the atmospheric neutrino spectra that are currently used to study the data of high energy neutrino telescopes are calculated using the assumption 17 that the primary CR spectra are simple power laws between the sub-TeV hardening and the Knee. Introducing the softening feature for primary protons and extrapolating with a constant slope to high energy (up to the Knee) results in neutrino spectra that are lower and softer. A too large reduction of the calculated $\nu$ flux could in fact be in tension with the IceCube observations.

A quantitative study of this problem requires the modeling of the spectra not only for protons but also for all other nuclei that give smaller but important contributions to the atmospheric neutrino fluxes, and will not be developed here. Present and future observations of the neutrino fluxes can however be used to constraint the primary CR fluxes.

Developing an understanding of the astrophysical mechanisms that shape the Galactic CR spectra and generate the observed features is a challenging undertaking. The standard "text-book" models for the origin of the Galactic cosmic rays (see for example [27]) predict (for protons and primary nuclei) spectra that have a simple power law shape, (up to a maximum energy determined by the properties of the accelerators) with a spectral index that, in good approximation, is equal for all particle types. In these models the spectra are constructed assuming a "universal" CR source spectrum that has power law form with a slope $\alpha_{0}$ that is distorted by propagation effects with the rigidity dependence $\propto \rho^{\delta}$. The observable spectrum is then also of power law form with index $\alpha \simeq \alpha_{0}+\delta$. Such a scenario fails to describe the observed spectra.

After the discovery of the sub-TeV hardening by PAMELA, several authors have constructed models for the Galactic cosmic rays where the spectra do have such a spectral feature. Essentially all of these models are simple generalizations of the "standard" scenario described above. In the models the hardening is attributed to either the acceleration or the propagation of cosmic rays. In the first case one for example assume the existence of two different classes of accelerators that generate source spectra of different slopes and dominate the spectra below and above the hardening feature, or alternatively the existence of two regimes for acceleration at low and high energy. In the second case one can assume that the propagation effects have different rigidity dependences at low and high rigidity.

In all cases, the modified models predict that above the hardening the CR spectra continue with a constant slope up to the Knee. The discovery of the softening is therefore a surprise that requires the construction of new (and more complex) models.

In principle, to explain the presence of two (and not just one) spectral features it is still possible to adopt what is essentially the same "methodology" discussed above to explain a single hardening. For example one can assume the existence of three regimes for CR propagation in three different rigidity intervals, or introduce more classes of accelerators, or a combination of these effects. This approach can also be generalized to the presence of three of more spectral features, however this appears more and more unnatural and contrived.

Perhaps, especially if the data will reveal the existence of more spectral features, it could become interesting, or in fact necessary, to look for a different type of explanation.

It is interesting to note that the features in the CR spectra that we have discussed in this work can be considered as rather subtle distortions of a flux of power law shape, that are revealed only by high precision measurements. An attractive (even if quite "non-orthodox") possibility is that the CR spectra are generated by an ensemble of sources that release in interstellar space spectra that (in contrast with the "standard" scenario) do not have one (or only few) shapes, but have a large variety of shapes that individually are not of power law form. These components could then combine to form an average spectrum that has a nearly (but not exactly) power law form. The variations with energy of the spectral index of the observed CR fluxes could then reflect 
the existence of these multiple source components.

In this scenario the production of Galactic cosmic rays can be considered analogous to the formation of the time averaged spectrum of solar energetic particles, generated by the ensemble of solar flares [28]. The spectra of the individual flares have a large variety of spectral shapes, (that in general are not of power law form), while the sum of many flares results in a time averaged spectrum that can be reasonably well (but not exactly) described by a power law.

\section{References}

[1] H. S. Ahn et al., "Discrepant hardening observed in cosmic-ray elemental spectra," Astrophys. J. 714, L89 (2010) doi:10.1088/2041-8205/714/1/L89 arXiv:1004.1123 [astro-ph.HE]].

[2] O. Adriani et al. [PAMELA Collaboration], "PAMELA Measurements of Cosmic-ray Proton and Helium Spectra," Science 332, 69 (2011) doi:10.1126/science.1199172 arXiv:1103.4055 [astro-ph.HE]].

[3] M. Aguilar et al. [AMS Collaboration], "Precision Measurement of the Proton Flux in Primary Cosmic Rays from Rigidity 1 GV to 1.8 TV with the Alpha Magnetic Spectrometer on the International Space Station," Phys. Rev. Lett. 114, 171103 (2015). doi:10.1103/PhysRevLett.114.171103

[4] M. Aguilar et al. [AMS Collaboration], "Precision Measurement of the Helium Flux in Primary Cosmic Rays of Rigidities 1.9 GV to $3 \mathrm{TV}$ with the Alpha Magnetic Spectrometer on the International Space Station," Phys. Rev. Lett. 115, no. 21, 211101 (2015). doi:10.1103/PhysRevLett.115.211101

[5] A. D. Panov et al., "Energy Spectra of Abundant Nuclei of Primary Cosmic Rays from the Data of ATIC-2 Experiment: Final Results," Bull. Russ. Acad. Sci. Phys. 73, no. 5, 564 (2009) [Izv. Ross. Akad. Nauk Ser. Fiz. 73, 602 (2009)] doi:10.3103/S1062873809050098 [arXiv:1101.3246 [astro-ph.HE]].

[6] Y. S. Yoon et al., "Proton and Helium Spectra from the CREAM-III Flight," Astrophys. J. 839, no. 1, 5 (2017) doi:10.3847/1538-4357/aa68e4 arXiv:1704.02512 [astro-ph.HE]].

[7] O. Adriani et al., "Direct Measurement of the Cosmic-Ray Proton Spectrum from $50 \mathrm{GeV}$ to $10 \mathrm{TeV}$ with the Calorimetric Electron Telescope on the International Space Station," Phys. Rev. Lett. 122, no. 18, 181102 (2019) doi:10.1103/PhysRevLett.122.181102 arXiv:1905.04229 [astro-ph.HE]].

[8] E. Atkin et al., "New Universal Cosmic-Ray Knee near a Magnetic Rigidity of 10 TV with the NUCLEON Space Observatory," JETP Lett. 108, no. 1, 5 (2018) doi:10.1134/S0021364018130015 arXiv:1805.07119 [astro-ph.HE]].

[9] E. V. Atkin et al., "Energy Spectra of Cosmic-Ray Protons and Nuclei Measured in the NUCLEON Experiment Using a New Method," Astron. Rep. 63, no. 1, 66 (2019) [Astron. Zh. 96, no. 1, 75 (2019)]. doi:10.1134/S1063772919010013

[10] Q. An et al. [DAMPE Collaboration], Science Advances, Vol. 5, no. 9, eaax3793 (2019) doi:10.1126/sciadv.aax3793 arXiv:1909.12860 [astro-ph.HE]].

[11] P. Lipari, "Spectral features in the cosmic ray fluxes," Astropart. Phys. 97, 197 (2018) doi:10.1016/j.astropartphys.2017.11.008 arXiv:1707.02504 [astro-ph.HE]].

[12] M. Amenomori et al. [TIBET III Collaboration], "The All-particle spectrum of primary cosmic rays in the wide energy range from $10^{14} \mathrm{eV}$ to $10^{17} \mathrm{eV}$ observed with the Tibet-III air-shower array," Astrophys. J. 678, 1165 (2008) doi:10.1086/529514 arXiv:0801.1803 [hep-ex]].

[13] [IceCube Collaboration], "Cosmic Ray Spectrum and Composition from PeV to EeV Using 3 Years of Data From IceTop and IceCube," arXiv:1906.04317 [astro-ph.HE]. 
[14] T. K. Gaisser, T. Stanev and S. Tilav, Front. Phys. (Beijing) 8, 748 (2013) doi:10.1007/s11467-013-0319-7 arXiv:1303.3565 [astro-ph.HE]].

[15] T. Antoni et al. [KASCADE Collaboration], "KASCADE measurements of energy spectra for elemental groups of cosmic rays: Results and open problems," Astropart. Phys. 24, 1 (2005) doi:10.1016/j.astropartphys.2005.04.001 astro-ph/0505413.

[16] N. N. Kalmykov and S. S. Ostapchenko, "The Nucleus-nucleus interaction, nuclear fragmentation, and fluctuations of extensive air showers," Phys. Atom. Nucl. 56, 346 (1993) [Yad. Fiz. 56, no. 3, 105 (1993)].

[17] R. Engel, T. K. Gaisser, T. Stanev and P. Lipari, "Air Shower Calculations with the New Version of SIBYLL," in Proc. 26th Int. Cosmic Ray Conf. Salt Lake City (USA) 1 (1999) 415.

[18] H. Fesefeldt, "The Simulation of Hadronic Showers: Physics and Applications," PITHA-85-02.

[19] W. D. Apel et al., "KASCADE-Grande measurements of energy spectra for elemental groups of cosmic rays," Astropart. Phys. 47, 54 (2013) doi:10.1016/j.astropartphys.2013.06.004 arXiv:1306.6283 [astroph.HE]].

[20] S. Ostapchenko, "QGSJET-II: Towards reliable description of very high energy hadronic interactions," Nucl. Phys. Proc. Suppl. 151, 143 (2006) doi:10.1016/j.nuclphysbps.2005.07.026 hep-ph/0412332.

[21] G. Battistoni, S. Muraro, P. R. Sala, F. Cerutti, A. Ferrari, S. Roesler, A. Fasso and J. Ranft, AIP Conf. Proc. 896, no. 1, 31 (2007). doi:10.1063/1.2720455

[22] M. Aguilar et al. [AMS Collaboration], "Observation of the Identical Rigidity Dependence of He, C, and O Cosmic Rays at High Rigidities by the Alpha Magnetic Spectrometer on the International Space Station," Phys. Rev. Lett. 119, no. 25, 251101 (2017). doi:10.1103/PhysRevLett.119.251101

[23] R. Alfaro et al. [HAWC Collaboration], "All-particle cosmic ray energy spectrum measured by the HAWC experiment from 10 to 500 TeV," Phys. Rev. D 96, no. 12, 122001 (2017) doi:10.1103/PhysRevD.96.122001 arXiv:1710.00890 [astro-ph.HE]].

[24] B. Bartoli et al. [ARGO-YBJ Collaboration], Phys. Rev. D 85, 092005 (2012). doi:10.1103/PhysRevD.85.092005

[25] B. Bartoli et al. [ARGO-YBJ and LHAASO Collaborations], "Knee of the cosmic hydrogen and helium spectrum below $1 \mathrm{PeV}$ measured by ARGO-YBJ and a Cherenkov telescope of LHAASO," Phys. Rev. D 92, no. 9, 092005 (2015) doi:10.1103/PhysRevD.92.092005 arXiv:1502.03164 [astro-ph.HE]].

[26] X. Bai et al., "The Large High Altitude Air Shower Observatory (LHAASO) Science White Paper," arXiv:1905.02773 [astro-ph.HE].

[27] T. K. Gaisser, R. Engel and E. Resconi, "Cosmic Rays and Particle Physics" Cambridge University Press (2016). doi:10.1017/CBO9781139192194

[28] R.A. Mewaldt, C.M.S. Cohen, G.M. Mason, D.K. Haggerty, M.I. Desai "Long-Term Fluences of Solar Energetic Particles from H to Fe" Space Science Reviews, 130, 323 (2007) doi:10.1007/s11214-007-9200-8 


\begin{tabular}{|c|c|c|c|c|c|c|c|c|c|c|c|}
\hline $\begin{array}{l}\stackrel{\pi}{\pi} \\
\stackrel{\pi}{\pi} \\
\Xi\end{array}$ & $\begin{array}{l}\ddot{0} \\
0 \\
0 \\
H \\
0 \\
0 \\
i \\
ن\end{array}$ & $\begin{array}{l}+0 \\
0 \\
0 \\
+1 \\
1 \\
10 \\
i \\
i\end{array}$ & $\begin{array}{l}200 \\
0.0 \\
00 \\
+1 \\
\infty \\
\infty \\
i \\
i\end{array}$ & 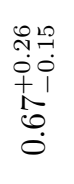 & 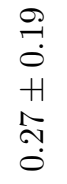 & $\begin{array}{l}m_{\infty} \\
+1 \\
0 \\
+\end{array}$ & $\begin{array}{l}\forall n \\
00 \\
+0 \\
+0 \\
20 \\
0 \\
0\end{array}$ & $\begin{array}{l}\text { Oे } \\
\stackrel{+}{0} \\
+1 \\
\stackrel{+}{+} \\
\stackrel{+}{+}\end{array}$ & 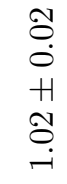 & $\begin{array}{l}20 \\
\dot{\varphi} \\
\mathscr{f}\end{array}$ & $\begin{array}{c}\infty \\
1 \\
\stackrel{\sim}{J}\end{array}$ \\
\hline 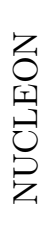 & 1 & 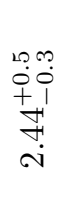 & $\begin{array}{l}20-1 \\
0.0 \\
+0 \\
01 \\
0 \\
0 \\
i\end{array}$ & 1 & 1 & $\begin{array}{l}00 \\
\infty \\
\infty \\
+1 \\
10 \\
0 \\
0\end{array}$ & 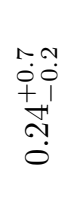 & । & 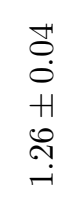 & $\ddot{\vec{\sigma}}$ & $\begin{array}{l}10 \\
1 \\
7\end{array}$ \\
\hline 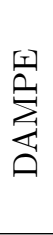 & $\begin{array}{l}20 \\
8 \\
0 \\
0 \\
H \\
0 \\
0 \\
1 \\
i \\
i\end{array}$ & $\begin{array}{l}\overrightarrow{0} \\
0 \\
0 \\
H \\
0 \\
0 \\
10 \\
ن \\
ن\end{array}$ & 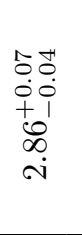 & 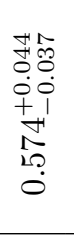 & 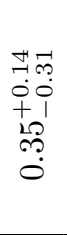 & 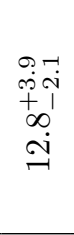 & 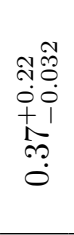 & $\begin{array}{l}8 \\
0 \\
0 \\
+1 \\
10 \\
20 \\
20 \\
20\end{array}$ & $\begin{array}{l}\mathcal{O} \\
\stackrel{0}{0} \\
H \\
10 \\
\stackrel{-1}{-1}\end{array}$ & $\stackrel{\vec{\theta}}{0}$ & $\begin{array}{l}\infty \\
1 \\
\end{array}$ \\
\hline 鼻 & $\begin{array}{l}\overrightarrow{0} \\
\dot{0} \\
H \\
\dot{H} \\
\overrightarrow{0} \\
\dot{\sigma}\end{array}$ & $\begin{array}{l}\overrightarrow{0} \\
0 \\
0 \\
H \\
20 \\
20 \\
0 \\
0\end{array}$ & 1 & $\begin{array}{l}80 \\
80 \\
0.0 \\
00 \\
+1 \\
20 \\
0 \\
0 \\
0 \\
0\end{array}$ & 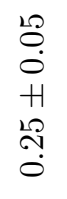 & 1 & 1 & 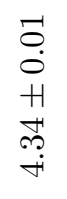 & $\begin{array}{l}92 \\
00 \\
00 \\
0+1 \\
01 \\
10 \\
0 \\
0 \\
0\end{array}$ & $\stackrel{\Re}{\stackrel{\leftrightarrow}{\sim}}$ & \begin{tabular}{c}
10 \\
1 \\
\hdashline \\
\end{tabular} \\
\hline$\sum_{\substack{|c| \\
\text { 空 }}}$ & 1 & $\begin{array}{l}\overrightarrow{0} \\
0 \\
0 \\
H \\
\infty \\
\infty \\
10 \\
ن\end{array}$ & 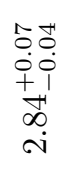 & 1 & 1 & $\begin{array}{c}100 \\
0 \\
0 \\
0\end{array}$ & $\begin{array}{l}100 \\
0 \\
+1 \\
0 \\
0\end{array}$ & 1 & \begin{tabular}{l}
$\vec{\sigma}$ \\
$\dot{+}$ \\
$H$ \\
\multirow{N}{*}{} \\
-
\end{tabular} & $\stackrel{\leftrightarrow}{\sim}$ & $\begin{array}{c}10 \\
1 \\
\text { I } \\
\text { I }\end{array}$ \\
\hline 导 & 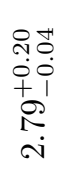 & 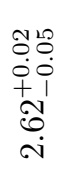 & 1 & 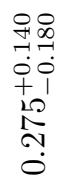 & 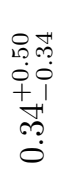 & 1 & 1 & 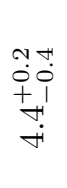 & 1 & & $\begin{array}{c}10 \\
1 \\
10 \\
-10\end{array}$ \\
\hline $\begin{array}{l}\text { Oे } \\
\text { की } \\
\sum_{4}\end{array}$ & 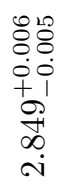 & 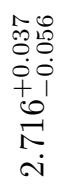 & 1 & 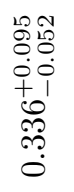 & 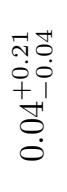 & 1 & 1 & $\begin{array}{l}\text { Oे } \\
\stackrel{0}{0} \\
+ \\
\text { I } \\
\stackrel{+}{+}\end{array}$ & 1 & $\stackrel{\leftrightarrow}{\leftrightarrow}$ & $\frac{10}{1}$ \\
\hline 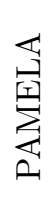 & $\begin{array}{l}\stackrel{9}{P} \\
\stackrel{0}{0} \\
0 \\
H \\
0 \\
0 \\
\infty \\
\infty \\
\text { i }\end{array}$ & 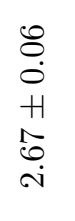 & 1 & 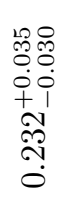 & 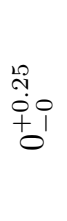 & 1 & 1 & $\begin{array}{l}\mathscr{8} \\
\dot{0} \\
+1 \\
\stackrel{0}{0} \\
\dot{+}\end{array}$ & 1 & & $\begin{array}{c}10 \\
1 \\
0 \\
0\end{array}$ \\
\hline & $\vec{\delta}$ & $\tilde{\delta}$ & $\mathscr{8}$ & 10요 & ३ & 迁 & ? & 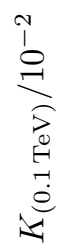 & 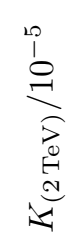 & 凊 & $z^{\frac{\vec{o}}{c}}$ \\
\hline
\end{tabular}




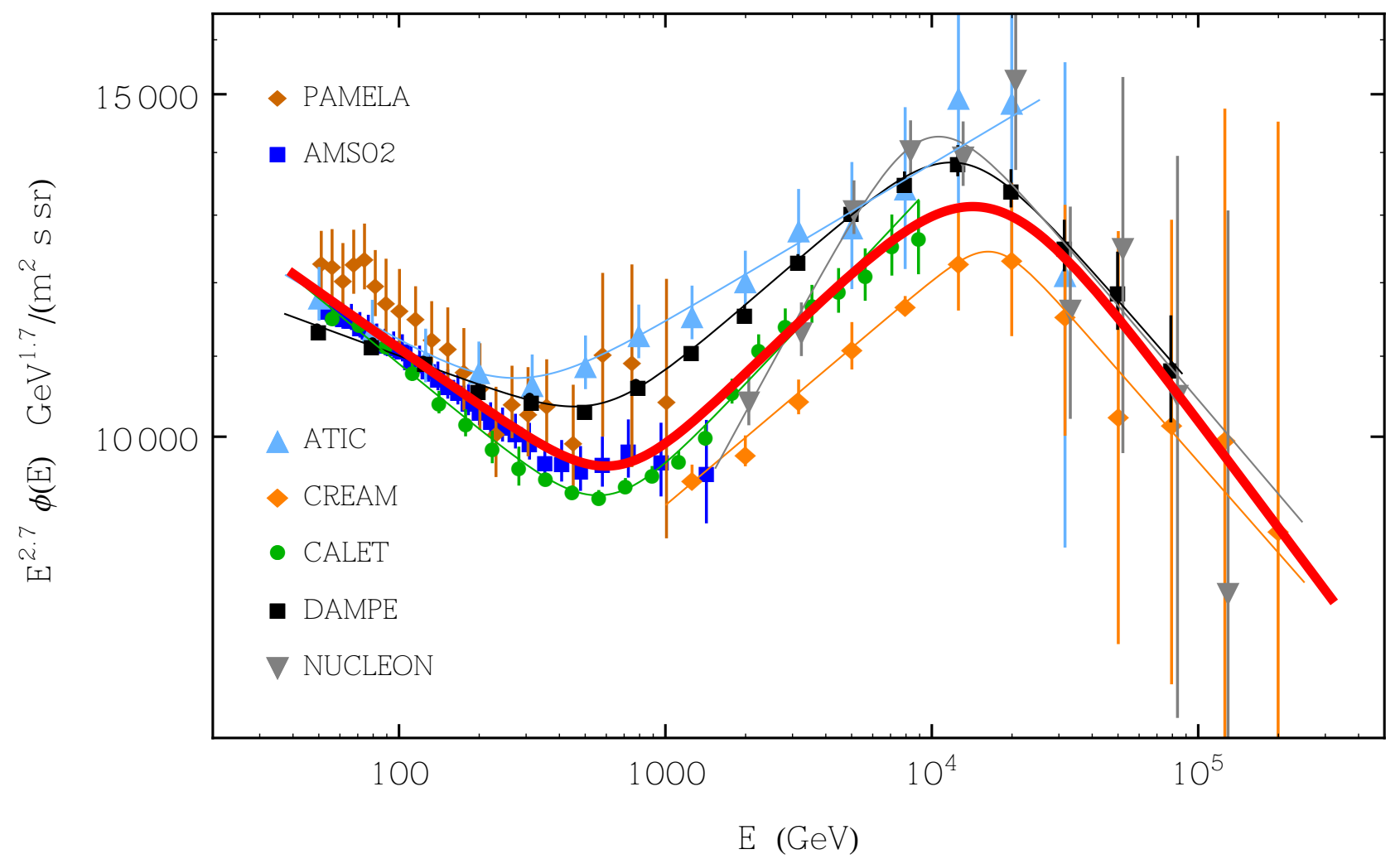

Figure 1: Direct measurements of the CR proton spectrum. The flux is shown in the form $E^{2.7} \phi(E)$ versus $E$ to enhance the visibility of the spectral features. The points are the data of PAMELA [2], AMS02 [4, ATIC [5], CREAM [6], CALET [7, DAMPE [10] and NUCLEON [8]. The thick (red) solid line is a fit of the combined data of all the experiments using the two-break expression 11. The thin lines are fits of the data of individual experiments. The parameters of all fits are listed in Table 1 . 


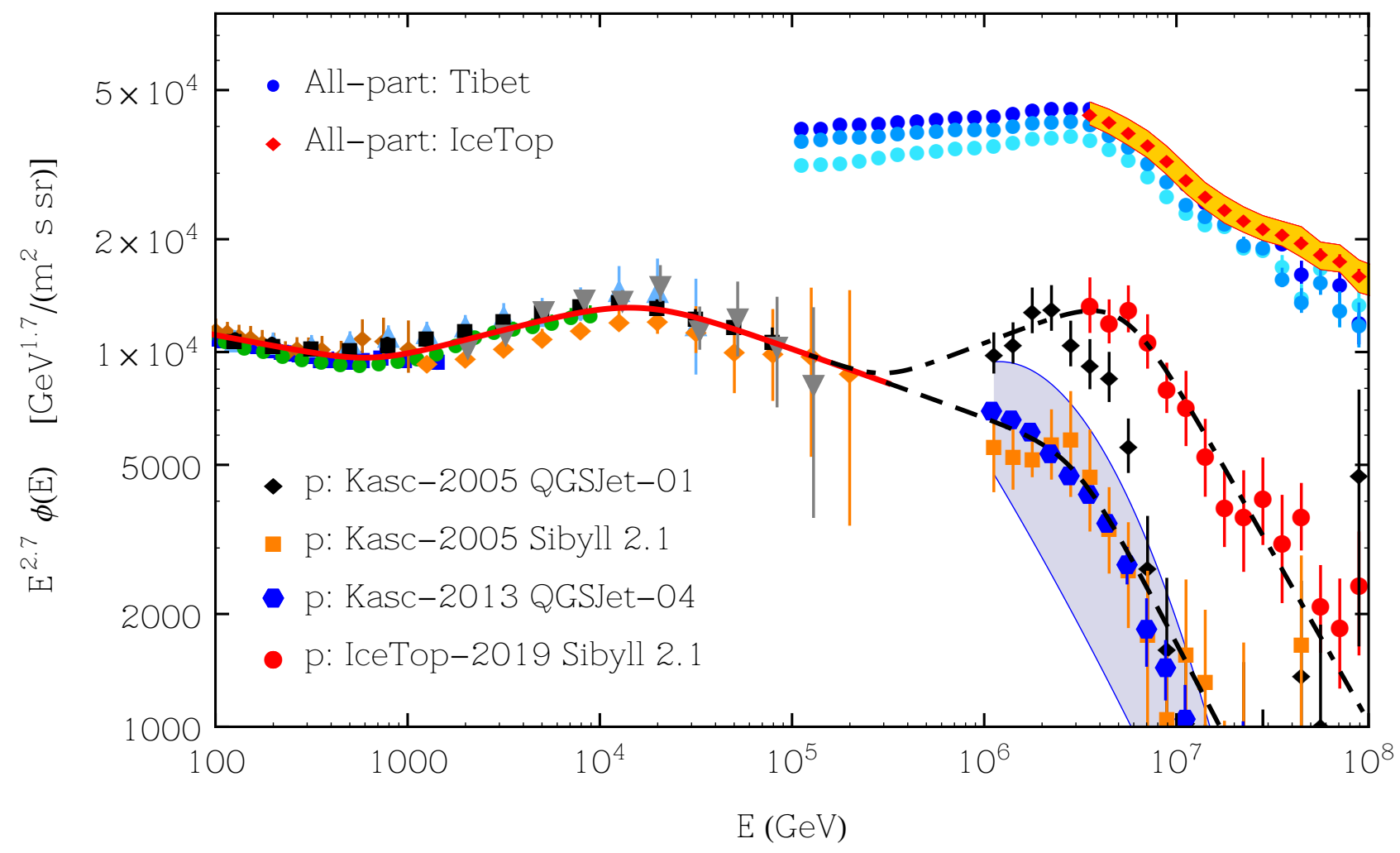

Figure 2: All-particle and proton spectra obtained by direct measurements and EAS observations. The all-particle data are by the Tibet experiment 12 (with three sets of data points obtained with different assumptions for the CR composition and shower development models), and by IceTop/IceCube [13] (with the shaded area indicating systematic uncertainties). For the proton direct measurements the symbols are identical to those in Fig. 1] The EAS proton spectra are by Kascade-2005 [15], Kascade-2013 [19] (with the shaded area indicating systematic uncertainties) and IceTop/IceCube-2019 13. The thick solid line is a fit to the direct measurements of the proton flux (with the parameters given in Table 1 1 . The dashed and dot-dashed lines are extrapolations to higher energy (see main text). 